\title{
Deep Brain Stimulation for Refractory Tinnitus: Pilot Study Protocol for a Randomized Double-Blinded Crossover Trial
}

\section{Gusta van Zwieten}

Maastricht University Hospital: Maastricht Universitair Medisch Centrum+

\section{Jana V P Devos}

Maastricht University Hospital: Maastricht Universitair Medisch Centrum+

\section{Sonja A Kotz}

Maastricht University Hospital: Maastricht Universitair Medisch Centrum+

Linda Ackermans

Maastricht University Hospital: Maastricht Universitair Medisch Centrum+

Pia Brinkmann

Maastricht University Hospital: Maastricht Universitair Medisch Centrum+

\section{Lobke Dauven}

Maastricht University Hospital: Maastricht Universitair Medisch Centrum+

\section{Erwin L J George}

Maastricht University Hospital: Maastricht Universitair Medisch Centrum+

\section{A. Miranda L Janssen}

Maastricht University Hospital: Maastricht Universitair Medisch Centrum+

\section{Bernd Kremer}

Maastricht University Hospital: Maastricht Universitair Medisch Centrum+

\section{Carsten Leue}

Maastricht University Hospital: Maastricht Universitair Medisch Centrum+

\section{Michael Schwartze}

Maastricht University Faculty of Health, Medicine and Life Sciences: Maastricht Universitair Medisch Centrum+

\section{Yasin Temel}

Maastricht University Hospital: Maastricht Universitair Medisch Centrum+

\section{Jasper V Smit}

Zuyderland Medical Centre Sittard-Geleen: Zuyderland Medisch Centrum Sittard-Geleen

Marcus L F Janssen ( $\sim$ m.janssen@maastrichtuniversity.nl )

Maastricht University Medical Center https://orcid.org/0000-0003-0030-7224

\section{Study Protocol}

Keywords: deep brain stimulation, tinnitus, MGB, auditory thalamus, randomized controlled trial

Posted Date: July 13th, 2021

DOI: https://doi.org/10.21203/rs.3.rs-692616/v1

License: (c) (1) This work is licensed under a Creative Commons Attribution 4.0 International License. Read Full License 


\section{Abstract}

Background: Chronic tinnitus can have an immense impact on quality of life. Despite recent treatment advances, many tinnitus patients remain refractory to them. Preclinical and clinical evidence suggest that deep brain stimulation (DBS) is as a promising treatment to suppress tinnitus. The thalamic medial geniculate body (MGB) takes a key position in the tinnitus network, shows pathophysiological hallmarks of tinnitus, and is readily accessible using stereotaxy. This study will evaluate the safety and therapeutic effects of DBS in the MGB in severe tinnitus sufferers.

Methods: Bilateral DBS of the MGB will be applied in six patients with severe and refractory tinnitus. A double-blinded, randomized $2 \times 2$ crossover design (stimulation ON and OFF) will be applied, followed by a period of six months open label follow-up. The primary focus is to assess safety and feasibility_(acceptability.). Secondary outcomes assess a potential treatment effect and include tinnitus severity_measured by the Tinnitus Functional Index (TFI), tinnitus loudness and distress, hearing, cognitive and psychological functions, quality of life, and neurophysiological characteristics.

Discussion: Whilst carefully balancing risks and benefits and taking ethical considerations into account, this study explores the safety and feasibility of DBS in severe refractory tinnitus, by extensive assessment of clinical and neurophysiological outcome measures. Additionally, important insights in the underlying mechanism of tinnitus and hearing function might be revealed.

Trial registration: ClinicalTrials.gov NCT03976908 (June 6, 2019)

\section{Background}

Tinnitus, commonly known as "ringing of the ears", is one of the largest health challenges in the world (1). According to a recent large survey, approximately $6.4 \%$ of Americans experience persistent tinnitus (2). Every tenth patient experiences a most extreme and debilitating form of tinnitus. Sleep deprivation, anxiety, and depression often accompany tinnitus and severely affect the patient's quality of life (3-5). In turn, this places a huge burden on society, healthcare costs, and decreases productivity (6).

Subjective tinnitus has a multifactorial origin with heterogeneous patient profiles, which makes it a highly complex condition. The absence of an underlying medical cause in most cases leaves many patients without an available curative evidence-based treatment $(7,8)$. Tinnitus combined with sensorineural hearing loss might benefit from hearing aids. However, somewhere between $22 \%$ and $80 \%$ of affected patients are adequately served by using hearing aids $(9,10)$. Current clinical practice primarily aims at reducing the impact of tinnitus by providing psychoeducation and improving coping strategies via various psychological interventions $(11,12)$.

The etiological and pathophysiological mechanisms of subjective tinnitus are complex and not fully understood. Many investigators feel that in nearly all tinnitus cases, there is some degree of cochlear impairment, leading to diminished auditory nerve activity reaching the cochlear nuclei (13). Much evidence implicates the head and neck somatosensory system as a separate major factor in the development of tinnitus. It is likely that most tinnitus develops as a result of CNS interactions between these two systems (14). According to current theories, tinnitus is associated with increased neural activity in auditory cortices, possibly resulting from maladaptive gating $(15,16)$ and/or increase in central gain $(17)$. Specific neural correlates described in tinnitus models are enhanced neuronal synchrony, increased spontaneous firing, and changes in tonotopic organization (18). The medial geniculate body (MGB) of the thalamus is a major relay and gateway between midbrain and cortex, and a core structure in tinnitus pathophysiology $(19,20)$. Preclinically, at multiple levels of the central auditory pathway from cochlear nuclei to auditory cortex and including the MGB, tinnitus related neuronal activity is similar to subthalamic nucleus activity in Parkinson's disease, i.e., enhanced spontaneous activity and burst firing (21-24). The primary role of auditory thalamic neurons is to actively and dynamically shape neural representations of information and to control which information reaches the cerebral cortex (25). Furthermore, integration of auditory and limbic information occurs within the MGB (26). Connected limbic structures, such as the amygdala and nucleus accumbens, are related to emotional and 
attentional symptoms of tinnitus (27). Hence, the MGB acts as a central hub in involved tinnitus networks, which makes it a promising structure for neuromodulatory approaches.

A commonly applied neuromodulation technique is deep brain stimulation (DBS). This therapy has been widely used in neurologic and neuropsychiatric disorders such as Parkinson's disease. DBS is generally applied using high frequency stimulation $(>100 \mathrm{~Hz})$, to disrupt pathological neuronal activity and oscillations $(28,29)$. Hypothetically, this results in alteration of tinnitus perception and related distress. Further, patients, treated with DBS for a movement disorder sometimes also suffered from tinnitus. DBS of non-auditory structures in these patients led to diminished or completely suppressed tinnitus (30-32). Moreover, preclinical studies support the beneficial effects of DBS on tinnitus when applied in auditory brain areas. A reduction in tinnitus-like behavior was shown when DBS was applied in several structures along the classical auditory pathway, including the dorsal cochlear nucleus $(33,34)$, inferior colliculus (35), and MGB (36). Importantly, no undesired sideeffects occurred. DBS of the MGB did not lead to anxiety or disturbed locomotor activity. DBS of the inferior colliculi did not cause any detectable hearing impairment (37). Therefore, DBS seems to be a promising treatment for severe, refractory tinnitus (38-40). The MGB is a preferred target area next to other auditory subcortical structures (39), as the auditory thalamus is readily accessible in stereotactic surgery. Consequently, targeting the auditory thalamus bears smaller surgical risks and complications such as bleeding and potential neurological deficit. Currently, MGB DBS has not been applied in humans. The majority of patients with tinnitus can be treated with non-invasive methods, and only a small number of patients can be considered as a candidate for DBS.

The primary objective of the proposed study is to assess the safety and feasibility (acceptability) of bilateral MGB DBS in severe tinnitus. Patients with severe tinnitus who are refractory to the standard treatment program will be included. Secondary outcomes will provide data on the potential efficacy of MGB DBS on tinnitus severity (Tinnitus Functional Index), tinnitus loudness, and distress (Visual Analogue Scales). Additionally, hearing (audiometry), cognition (neuropsychological test battery), quality of life, and psychological functioning (questionnaires) will be assessed. Furthermore, electrophysiological data will assess fundamental aspects of auditory function and tinnitus pathophysiology. After successful evaluation of the primary and secondary outcomes in this pilot study, MGB DBS could potentially be further developed as a novel treatment option in severe, refractory tinnitus.

\section{Methods}

\section{Study design}

This pilot study uses a double-blind $2 \times 2$ cross-over design in which MGB DBS will be compared to no stimulation (Fig. 1).

\section{Setting}

This study will be carried out at Maastricht University Medical Center (MUMC+) in Maastricht, the Netherlands. MUMC + is an expertise center for tinnitus, providing integrated multidisciplinary diagnostics and rehabilitation for a wide range of tinnitus patients. The Ear, Nose, and Throat department has long-standing clinical expertise and experience with developing neuromodulative therapies for tinnitus such as intracochlear devices (41). The neurosurgery department has substantial clinical and preclinical expertise in DBS. In addition to a preclinical DBS research line (42), it is well equipped to conduct clinical trials for new indications of DBS therapy such as Gilles de la Tourette syndrome (43). Acquisition of neurophysiological data, both intra- and postoperatively is standard practice and is used to unravel neural mechanisms (44).

The study will be conducted according to the principles of the Declaration of Helsinki (Version 10, 2013) and in accordance with the Medical Research Involving Human Subjects Act (WMO). This study complied with the CONsolidated Standards Of Reporting Trials (CONSORT) extension statement. Ethics approval was obtained by the institutional review board. Results will be published in an international peer reviewed scientific journal and presented at scientific meetings.

\section{Recruitment and consent}


Patients are eligible to enroll if they meet the in- and exclusion criteria as outlined in Table 1. All patients will be evaluated and selected by a multidisciplinary team of specialists (otolaryngologists, audiologists, neurosurgeons, neurologists, psychiatrists and psychologists). Diagnostics and treatment are in accordance with national tinnitus guidelines (45). Based on the Dutch tinnitus guidelines, the tinnitus questionnaire (TQ) is used to determine tinnitus severity (46). Patients suffer severely (TQ score $\geq 47$ ), and are refractory to available treatments including cognitive behavioral therapy and hearing aids in case of hearing loss.

Table 1

Eligibility criteria. TQ, Tinnitus Questionnaire; DBS, Deep Brain Stimulation; Cl, Cochlear Implant; ABI, Auditory Brainstem Implant.

Inclusion criteria Exclusion criteria

- Medically refractory tinnitus *

- Age 18-69 years

- Experiencing tinnitus that is non-pulsatile and uni- or bilateral

- Severe tinnitus (TQ score $\geq 47$ )

- Tinnitus, which is chronic (present $\geq$ two years)

and stable (not intermittent $\geq$ one year)

- Average pure tone thresholds for 1, 2 and $4 \mathrm{kHz}<$ $60 \mathrm{~dB}$ for each ear

- Willingness to participate in the study
- Anatomic cause of tinnitus (e.g., vestibular schwannoma, tumor, middle-ear pathology, temporal mandibular disorder)

- DSM-V psychiatric disorders, other than depression or anxiety disorder

- Depression or anxiety disorder, manifestation before tinnitus onset

- Cognitive impairment or coping problems

- Active otologic diseases

- Pregnancy or breast-feeding

- Active suicidal thoughts or recent attempts

- Life expectancy lower than two years

- Implantable electronic devices that potentially interfere with DBS ( $\mathrm{Cl}, \mathrm{ABI}$, cortical implant)

- General contra-indications for MRI or surgery

* Patient does not respond to available tinnitus treatments (e.g., sound enrichment and cognitive behavioral therapy) and is thoroughly evaluated by the multidisciplinary tinnitus team in MUMC+.

Patients will be recruited at the outpatient clinic of the Ear Nose Throat department. If patients give permission, they receive an information brochure. Two weeks after, researchers will contact the patients to plan a face-to-face meeting. During this meeting, a full understanding of the study protocol is ensured and additional questions are answered. When a patient needs more time to decide, the investigator plans a follow-up appointment after a few weeks. If a patient agrees to participate in the study, informed consent will be signed by the patient and investigator. If the patient meets the criteria, a second outpatient visit will be planned. During this visit, an intake interview will be conducted by one of the researchers, followed by a consultation with both a psychiatrist and a neurosurgeon. Then, the multidisciplinary team will form a collective decision on inclusion or exclusion. Following a positive decision of the multidisciplinary team, standard clinical workup for DBS surgery will follow. This includes conducting a brain scan and general blood examination. If inclusion criteria are still met, final inclusion will follow. Patients can leave the study at any time for any reason without any consequences. The investigators can decide to withdraw a subject from the study for urgent medical reasons.

\section{Outcomes}

The time frame and methods of data acquisition are displayed in Fig. 1 and Table 2, respectively. 
Table 2

Schedule of assessments and procedures. T0 is at inclusion. T1 is at the end of the first 6-week treatment period (stimulation

ON or OFF). T2 is at the end of the second 6-week treatment period (stimulation OFF or ON). T3 is at the end of the third

treatment period of 6 months stimulation ON. MRI, Magnetic Resonance Imaging; CT, Computer Tomography; TFI, Tinnitus

Functional Index; VAS, Visual Analogue Scale; ABR, Auditory Brainstem Responses; EEG, Electroencephalography; LFP, Local

Field Potentials.

$\begin{aligned} & \text { Inclusion Surgery Recovery } \\ & \text { (T0) }\end{aligned}$
Optimization Period washout

(T1) (T2)

(T3)

\section{Visits and procedures}

\begin{tabular}{|lllll|}
\hline Outpatient visit & 2 & 6 & 2 & 2 \\
\hline $\begin{array}{l}\text { Anesthesiology } \\
\text { screening }\end{array}$ & $\cdot$ & & & \\
\hline
\end{tabular}

MRI

CT

\section{Outcome measures}

Tinnitus severity $\quad$.

TFI

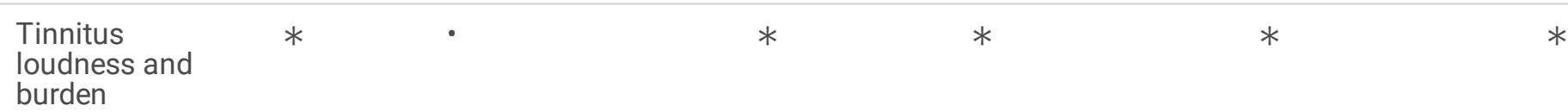

VAS

Hearing function

Audiometry

ABR

Cognitive

functioning**

Psychological

functioning**

Neurophysiology

EEG

LFP

* Last week of the period, 3 random times per day

${ }^{* *}$ Cognitive functioning tests include Boston Naming Test, Verbal and letter fluency, 15 Word Test, Trail Making Test and

Stroop Color-Word Test. Psychological functioning is assessed using 36-Item Short Form Health Survey (SF-36), Beck

Depression Inventory II (BDI-II), Beck Anxiety Inventory (BAI), and Hospital Anxiety and Depression Scale (HADS).

\section{Primary outcomes}

The primary focus of this pilot study is to assess safety and feasibility. Safety will be assessed by reporting the rate and grade of all surgical and stimulation induced adverse events in the study population during the study period. Feasibility will be assessed in terms of acceptability of the intervention, by taking qualitative interviews at all major timepoints (T0, T1, T2, T3), and by comparing satisfaction during sham stimulation and DBS.

\section{Secondary outcomes}


The secondary outcomes are meant to estimate a potential treatment effect of MGB DBS on tinnitus severity, hearing function, depression, anxiety, cognitive function, and quality of life. The small sample size may hinder a statistically significant outcome. Furthermore, changes in neuronal activity will be assessed by comparing electrophysiological measurements during sham stimulation and DBS.

1. Tinnitus severity will be assessed with the Tinnitus Functional Index (TFI) (47). The TFI is a validated self-report questionnaire that measures overall severity of tinnitus and provides coverage of multiple tinnitus severity domains. This questionnaire is the most appropriate responsive measure of treatment-related change. The TFI is translated and validated for Dutch native speakers (48).

2. Tinnitus loudness and burden will be measured by VAS. This will be done three times daily within a week, which is repeated four times during the study. Furthermore, these VAS-scores will be used to assess the effect of stimulation on tinnitus during surgery. VAS ratings for tinnitus loudness and burden are often used in both clinical practice and experimental and descriptive research as a measure of subjective symptoms (49). Both scales have been shown to correlate with the scores on Tinnitus Questionnaires (50).

3. Hearing function will be assessed with pure-tone and speech audiometry. These are clinical standard audiometric tests. Furthermore, subjective hearing will be evaluated using narratives.

4. Cognitive functioning will be measured using a validated test battery for standard DBS care. These include the Boston Naming Test, Verbal Fluency, Letter Fluency, 15 Words Test, Trail Making Test part A and B, and the Stroop Color-Word Test.

5. Quality of life and psychological functioning will be assessed by the following psychological questionnaires: 36-Item Short Form Health Survey (SF-36), Beck Depression Inventory II (BDI-II), Beck Anxiety Inventory (BAI), and Hospital Anxiety and Depression Scale (HADS).

6. Neurophysiological measurements: electrophysiological data and auditory brainstem responses will be recorded before and after surgery (T0 and recovery) and at the end of the treatment periods I and II (T1 and T2). Furthermore, local field potentials (LFP) will be recorded during surgery and before implantation of the pulse generator. Details are described under 'Neurophysiological assessments'.

Dependent on the results of the primary and secondary outcomes, future implementation procedures might be adapted or in case of adverse events, terminated. These adaptations depend not only on the narratives of the individual participants, but also on expert judgement.

\section{Intervention}

\section{Implantation of DBS electrodes and internal pulse generator}

A two-staged surgery will be performed. First, bilateral DBS electrodes will be implanted. Following a standard stereotactic surgical procedure, DBS electrodes will be inserted in the MGB of the thalamus and monitored by radiological and electrophysiological measures. The placement will be conducted under local anesthesia. A CT cerebrum will be carried out and fused with a pre-operative MRI in order to plan the exact trajectory. First, a micro-electrode (InoMed, Emmendingen, Germany) will be inserted. Neurophysiological recordings will be performed in 0.5 to $1 \mathrm{~mm}$ steps from $10 \mathrm{~mm}$ above and maximally 5 $\mathrm{mm}$ below the target with a multi-channel system (InoMed, Emmendingen, Germany) (51). Simultaneously, at each step, a sequence of auditory stimuli will be presented, which is designed based on known signal processing characteristics of the thalamus (52) and to maximize the likelihood of evoking a reliable responses. The amplitude of these responses relative to spontaneous activity will be used to confirm electrode placement. After the identification of the ventral and dorsal border of the MGB, test stimulation will be applied using $130 \mathrm{~Hz}$, pulse width of $120 \mu$ s and a current starting from $0.5 \mathrm{~V}$ up to $5 \mathrm{~V}$, or until undesired side-effects occur. Stimulation amplitude will be adjusted stepwise. The patient will be asked repeatedly to rate the loudness and burden of the tinnitus sound using VAS. Furthermore, the neurologist will test for undesired side-effects. The stimulation electrode will be placed once an optimal effect and acceptable or absent side-effects are reached. After placement and fixation of both stimulation electrodes (Medtronic, model 3389) the stereotactic frame will be removed and electrodes will be externalized to enable the recording of LFPs postoperatively. 
After surgery, the patient will be transferred to a neurosurgical medium care unit. On the second post-operative day a CT cerebrum will be made to confirm the definite electrode positioning. After one or two days the pulse generator (Medtronic, Activa PC model 37601) will be subcutaneously placed under general anesthesia and the electrodes will be connected. One or two days postoperative, the patient can be discharged. After the end of the trial, follow-up as standard DBS care will be provided.

\section{Stimulation parameters}

The following stimulation parameters will be used as minimum and maximum values during the optimization period to determine optimal stimulation parameters: stimulation frequency $(2-200 \mathrm{~Hz})$, pulse width $(60-450 \mu \mathrm{s})$, and voltage (starting from 0 to a maximum of $5 \mathrm{~V}$ ). In case of stimulation induced side-effects, bilateral stimulation can be adapted to unilateral stimulation. During treatment, patients will visit the outpatient clinic weekly. Following the treatment episode, there will be a one-week period for washout of a possible residual therapeutic effect.

\section{Neurophysiological assessments}

EEG will be recorded with surface electrodes applied to the scalp in accordance with the $10-20$ international system standard. Just before implantation of the pulse generator, cables are externalized, which enables simultaneous recordings of LFPs from the depth electrodes in the MGB.

For each session, several recordings with and without auditory stimulation will be obtained. In the first phase, resting-state activity will be recorded with eyes open and eyes closed. These initial recordings serve to establish baselines for taskindependent neural oscillatory activity, network activity, and coherence (assessed on the basis of dominant spontaneous lowfrequency oscillations and their variability). In the second phase, auditory brainstem responses will be recorded using a standard protocol to probe auditory brainstem function before and after surgery. In the third phase, activity will be recorded during experimental auditory stimulation. The respective measures will allow assessing of basic characteristics of auditory function in general, and sensory gating in particular (i.e., adaptive filtering based on predictable feature-based and temporal information) in accordance with the adopted model. These measures have been previously obtained via surface EEG recordings in humans $(53,54)$ and preliminary data show comparable responses recorded from the thalamus in the animal model.

Taken together, these measures allow assessing fundamental signal coding (linear vs. event-related), time-course (temporal relation of thalamic and surface responses), and functional (deviance processing, regularity, predictability, gating) aspects of auditory function. Dysfunctional processing would correspondingly be indexed by desynchronization, lack of suppression effects, temporal decoupling (reduced correlation between thalamic and surface responses), and overall high variability.

\section{Randomization and blinding}

Randomization will be performed directly after the period of stimulation optimization by an independent institution, the Clinical Trial Center Maastricht (CTCM). Patients will be randomly assigned to one of the two study groups. The investigator who adjusts DBS parameters cannot be blinded. All other investigators and the patient are blinded to the study group. In case the patients are considered unblinded due to the nature of the stimulation the protocol will be carried out as planned. Only in the case of medical concerns, the patient and investigators will be unblinded in order to provide the care needed.

\section{Data collection and management}

Data handling will be organized according to the "FAIR guiding principles for scientific data management and stewardship" (55). This will be done in cooperation with the CTCM, which is a local center that facilitates human research. Data will be collected by data entry in Castor EDC; a cloud-based, password protected data management system providing a back-up on a secured server. Audit trailing provides basic data to backtrack a file to its origin. Paper versions of questionnaires will be kept in a locked closet in the research office. All data will be anonymously coded. The key will be available to the project leader only. Data collection is monitored by the CTCM; a specific monitor is appointed to the study who will follow-up the progress and adherence to the protocol. The monitor will perform periodical checks. A data safety monitoring board, comprising of a 
statistician and two neurologists, is instituted which periodically reviews and evaluates the accumulated study data concerning participant safety, study conduct, progress, and efficacy. The data safety monitoring board receives and reviews the progress and acquired data of this trial and provides the research team with advice on the conduct of the trial.

\section{Statistical analyses}

The safety and feasibility outcomes will be reported descriptively and narratively. Descriptive statistics with $95 \%$ confidence intervals will be used to present preliminary data of secondary outcomes such as tinnitus (severity, loudness and distress), anxiety, depression, hearing function, quality of life, and cognitive functioning. These data will provide some insight into population characteristics and might indicate potential changes in mean scores between the intervention and sham stimulation.

\section{Sample size}

As this is a pilot study, no formal sample size calculation was performed. Based on previous first-in-human DBS studies (43, $56,57)$, we expect to adequately address the safety and the proof of concept purpose of the study by including six patients. Furthermore, this number of patients will enable collection of preliminary results that will provide meaningful information about differences between the intervention and sham stimulation. In case of the withdrawal of a patient or in case of incomplete data there will be no replacement of individual participants.

\section{Patient and public involvement}

Patients from the Dutch tinnitus support group were involved in the development of this protocol. During information meetings, aspects of the study were discussed such as feasibility and eligibility criteria. Furthermore, patients were involved in the development of the patient information.

\section{Discussion}

One of the main ethical considerations in this study is balancing risks and benefits. The potential benefit of the intervention is a reduction of tinnitus loudness and tinnitus burden. Risks of this minimally invasive and reversible form of functional neurosurgery are surgery related complications (e.g., cerebral haemorrhage or infarction, CSF leak, seizures, meningitis or encephalitis), hardware failure (e.g., lead rupture, extension fibrosis, device migration) and stimulation related side-effects. These latter effects are unknown as clinical MGB DBS has not been performed before. The function of the MGB is primarily hearing thus other side-effects than hearing-related effects are less likely. Animal studies did not show hearing loss, anxiety or locomotion related side-effects in DBS in subcortical auditory structures $(33,36,37)$. We also reviewed possible side-effects based on the brain structures surrounding the MGB. The MGB is located posterior to the subthalamic nucleus. The MGB is $8 \mathrm{~mm}$ wide, $6 \mathrm{~mm}$ long and $6.5 \mathrm{~mm}$ high with its largest volume at $-3.5 \mathrm{~mm}$ from the AC-PC plane. Considering the relatively large size of the MGB, current spread outside the MGB is unlikely if the DBS electrode is positioned in the center. Neighboring structures of the MGB are structures similar to other commonly used stimulation targets. Current spread to the anterior side of the MGB could result in internal capsule effects. These side effects are known from STN DBS treatment for dystonia and Parkinson's disease. The possible side effects of internal capsule stimulation are dysarthria, muscle contractions, and gaze paresis. Posterior to the MGB the ventricle can be found. Possible side effects are known from ANT DBS which has also a border at the ventricle. Antero-medially the sensory thalamus is located which is targeted when performing DBS to treat pain. More medially the fields of Forel are located. Possible side effects are known from STN DBS in which current spread also may occur to the fields of Forel which can result in disturbances of speech, postural stability and gait. Laterally to the MGB the optic tract is located. We know from GPi DBS that stimulation of the optic tract elicits phosphenes. All these side effects from current spread to surrounding regions are elicited by stimulation and thus reversible. Taking this together, MGB DBS could induce changes in auditory sensation, and there is a slight risk for side-effects due to current spread. In case of undesired sideeffects, stimulation parameters can be adapted or stimulation can be turned off. The principal investigator will immediately inform the data safety monitoring board and the medical ethical committee in case of serious side-effect. 
In relation to correctly and carefully evaluate risks and benefits, patient recruitment and extensive informed consent are crucial steps. The primary inclusion criterion is that patients need to be refractory to current treatment (e.g., cognitive behavioral therapy); second, sufficient hearing is required and patients cannot be candidates for cochlear implants. Last, motivation and written informed consent of patients to undergo DBS for tinnitus is essential. A questionnaire study showed that about $20 \%$ of tinnitus patients is willing to undergo DBS surgery in case of a $50 \%$ chance of successful treatment (58). Further, patients would be willing to pay 20 times their monthly income to be treated. Most patients would accept a risk of mild side-effects, and almost half of patients would accept a risk of severe side effects (58). A caveat in patient selection is that desperate patients might see this experimental treatment as a last resort and rush through the informed consent (59). These circumstances make this group of patients vulnerable and inclusion should carefully be contemplated when informed consent is discussed. Included patients will undertake a large effort by undergoing a surgical treatment that could be perceived as a last remedy. This could potentially lead to a bias based on effort justification. By using a randomized double blinded design, we aim to minimize this bias. An additional limitation is that generalizability to the target group of severely affected tinnitus patients might be challenging due to the heterogeneous nature of tinnitus and the small sample size of this pilot feasibility trial. Still, by carefully evaluating safety and feasibility in this pilot study, we will be able to determine how MGB DBS is received by participants, and optimize a follow-up study in terms of patient support, patient inclusion, surgical procedure and choice of stimulation parameters.

\section{Abbreviations}

Deep brain stimulation (DBS)

Medial geniculate body (MGB)

Tinnitus Functional Index (TFI)

Visual Analogue Scales (VAS)

Maastricht University Medical Center (MUMC+)

Tinnitus questionnaire (TQ)

36-Item Short Form Health Survey (SF-36)

Beck Depression Inventory II (BDI-II)

Beck Anxiety Inventory (BAl)

Hospital Anxiety and Depression Scale (HADS)

Local field potentials (LFP)

Clinical Trial Center Maastricht (CTCM)

\section{Declarations}

\section{Trial status}

Recruitment starts in 2020 and last follow-up is estimated to be completed in 2023.

\section{Contact information}

For public queries:

Drs. J.V.P. Devos

Page 9/14 
P.O. Box 616

6200 MD Maastricht, The Netherlands

Jana.devos@maastrichtuniversity.nl

$0031(0) 433881174$

For scientific queries $(\mathrm{PI})$ :

Dr. M.L.F. Jansssen

Medisch Universitair Ziekenhuis Maastricht

Neurologie

P. Debyelaan 25

6229 HX Maastricht, The Netherlands

m.janssen@maastrichtuniversity.nl

$0031(0) 624984487$

Ethics approval and consent to participate: Ethics approval was obtained by the institutional review board METC azM/UM (reference number NL67027.068.18 / METC 18-063 version 0.4 dated 06-08-2019). Written, informed consent to participate will be obtained from all participants. Results will be published in a peer reviewed scientific journal and presented at scientific meetings.

Consent for publication: Not applicable.

Availability of data and materials: Data can only be viewed by the investigators, IGJ and monitors.

Competing interests: There are no conflicts of interests to report.

Funding: The MUMC+ is the sponsor of the study. The investigators are responsible for study design; collection, management, analysis, and interpretation of data; writing of the report; and the decision to submit the report for publication. No funding from any commercial party is obtained to conduct this study.

Maastricht UMC+

Postbus 5800

6202 AZ Maastricht, NL

Author contributions: All authors contributed to the overall study design and specific methodologies. JS and MJ are leading the trial coordination and helped to conceive the project. GZ, JD, JS and MJ produced the detailed protocol, with input from LA, PB, LD, EG, AJ, BK, CL, and YT. SK and MS designed the neurophysiological assessment of the study. GZ and JD drafted the manuscript with assistance from SK, JS, and MJ. All authors have critically read, contributed with inputs and revisions, and approved the final manuscript.

Acknowledgements: Not applicable.

\section{References}


1. Henry JA, Dennis KC, Schechter MA. General review of tinnitus: prevalence, mechanisms, effects, and management. J Speech Lang Hear Res. 2005;48(5):1204-35.

2. American National Health. and Nutrition Examination Survey, Audiometry section (AUQ_G). 2011-2012.

3. Andersson G, Freijd A, Baguley DM, Idrizbegovic E. Tinnitus distress, anxiety, depression, and hearing problems among cochlear implant patients with tinnitus. J Am Acad Audiol. 2009;20(5):315-9.

4. Langguth $B$, Landgrebe M, Kleinjung T, Sand GP, Hajak G. Tinnitus and depression. World J Biol Psychiatry. 2011;12(7):489-500.

5. Zoger S, Svedlund J, Holgers KM. Relationship between tinnitus severity and psychiatric disorders. Psychosomatics. 2006;47(4):282-8.

6. Maes IH, Cima RF, Vlaeyen JW, Anteunis LJ, Joore MA. Tinnitus: a cost study. Ear Hear. 2013;34(4):508-14.

7. Langguth B, Kreuzer PM, Kleinjung T, De Ridder D. Tinnitus: causes and clinical management. Lancet Neurol. 2013;12(9):920-30.

8. Cima RFF, Mazurek B, Haider H, Kikidis D, Lapira A, Norena A, et al. A multidisciplinary European guideline for tinnitus: diagnostics, assessment, and treatment. HNO. 2019;67(Suppl 1):10-42.

9. Kochkin S, Tyler R. Tinnitus treatment and the effectiveness of hearing aids: hearing care professional perceptions. Hearing Review. 2008;15(13):14-8.

10. Trotter MI, Donaldson I. Hearing aids and tinnitus therapy: a 25-year experience. J Laryngol Otol. 2008;122(10):1052-6.

11. Jastreboff PJ. Tinnitus retraining therapy. Br J Audiol. 1999;33(1):68-70.

12. Cima RF, Andersson G, Schmidt CJ, Henry JA. Cognitive-behavioral treatments for tinnitus: a review of the literature. J Am Acad Audiol. 2014;25(1):29-61.

13. Eggermont JJ, Roberts LE. The neuroscience of tinnitus: understanding abnormal and normal auditory perception. Front Syst Neurosci. 2012;6:53.

14. Levine RA, Nam EC, Oron Y, Melcher JR. Evidence for a tinnitus subgroup responsive to somatosensory based treatment modalities. Prog Brain Res. 2007;166:195-207.

15. Durai M, Sanders M, Kobayashi K, Searchfield GD. Auditory Streaming and Prediction in Tinnitus Sufferers. Ear Hear. 2019;40(2):345-57.

16. Rauschecker JP, May ES, Maudoux A, Ploner M. Frontostriatal Gating of Tinnitus and Chronic Pain. Trends Cogn Sci. 2015;19(10):567-78.

17. Norena AJ. An integrative model of tinnitus based on a central gain controlling neural sensitivity. Neurosci Biobehav Rev. 2011;35(5):1089-109.

18. Eggermont JJ. Pathophysiology of tinnitus. Prog Brain Res. 2007;166:19-35.

19. Leaver AM, Renier L, Chevillet MA, Morgan S, Kim HJ, Rauschecker JP. Dysregulation of limbic and auditory networks in tinnitus. Neuron. 2011;69(1):33-43.

20. Rauschecker JP, Leaver AM, Muhlau M. Tuning out the noise: limbic-auditory interactions in tinnitus. Neuron. 2010;66(6):819-26.

21. Bergman H, Wichmann T, Karmon B, DeLong MR. The primate subthalamic nucleus. II. Neuronal activity in the MPTP model of parkinsonism. J Neurophysiol. 1994;72(2):507-20.

22. Benazzouz A, Breit S, Koudsie A, Pollak P, Krack P, Benabid AL. Intraoperative microrecordings of the subthalamic nucleus in Parkinson's disease. Mov Disord. 2002;17(Suppl 3):145-9.

23. Janssen ML, Zwartjes DG, Tan SK, Vlamings R, Jahanshahi A, Heida T, et al. Mild dopaminergic lesions are accompanied by robust changes in subthalamic nucleus activity. Neurosci Lett. 2012;508(2):101-5.

24. Kalappa BI, Brozoski TJ, Turner JG, Caspary DM. Single unit hyperactivity and bursting in the auditory thalamus of awake rats directly correlates with behavioural evidence of tinnitus. J Physiol. 2014;592(22):5065-78. 
25. Bartlett EL. The organization and physiology of the auditory thalamus and its role in processing acoustic features important for speech perception. Brain Lang. 2013;126(1):29-48.

26. Kraus KS, Canlon B. Neuronal connectivity and interactions between the auditory and limbic systems. Effects of noise and tinnitus. Hear Res. 2012;288(1-2):34-46.

27. De Ridder D, Vanneste S, Weisz N, Londero A, Schlee W, Elgoyhen AB, et al. An integrative model of auditory phantom perception: tinnitus as a unified percept of interacting separable subnetworks. Neurosci Biobehav Rev. 2014;44:16-32.

28. Grill WM, Snyder AN, Miocinovic S. Deep brain stimulation creates an informational lesion of the stimulated nucleus. Neuroreport. 2004;15(7):1137-40.

29. Chiken S, Nambu A. Disrupting neuronal transmission: mechanism of DBS? Front Syst Neurosci. 2014;8:33.

30. Cheung SW, Larson PS. Tinnitus modulation by deep brain stimulation in locus of caudate neurons (area LC). Neuroscience. 2010;169(4):1768-78.

31. Shi Y, Burchiel KJ, Anderson VC, Martin WH. Deep brain stimulation effects in patients with tinnitus. Otolaryngol Head Neck Surg. 2009;141(2):285-7.

32. Smit JV, Janssen ML, Engelhard M, de Bie RM, Schuurman PR, Contarino MF, et al. The impact of deep brain stimulation on tinnitus. Surg Neurol Int. 2016;7(Suppl 35):848-S54.

33. van Zwieten G, Jahanshahi A, van Erp ML, Temel Y, Stokroos RJ, Janssen MLF, et al. Alleviation of Tinnitus With HighFrequency Stimulation of the Dorsal Cochlear Nucleus: A Rodent Study. Trends Hear. 2019;23:2331216519835080.

34. Luo H, Zhang X, Nation J, Pace E, Lepczyk L, Zhang J. Tinnitus suppression by electrical stimulation of the rat dorsal cochlear nucleus. Neurosci Lett. 2012;522(1):16-20.

35. Smit JV, Janssen ML, van Zwieten G, Jahanshahi A, Temel Y, Stokroos RJ. Deep brain stimulation of the inferior colliculus in the rodent suppresses tinnitus. Brain Res. 2016.

36. van Zwieten G, Janssen MLF, Smit JV, Janssen AML, Roet M, Jahanshahi A, et al. Inhibition of Experimental Tinnitus With High Frequency Stimulation of the Rat Medial Geniculate Body. Neuromodulation. 2018.

37. Smit JV, Jahanshahi A, Janssen MLF, Stokroos RJ, Temel Y. Hearing assessment during deep brain stimulation of the central nucleus of the inferior colliculus and dentate cerebellar nucleus in rat. PeerJ. 2017;5:e3892.

38. Smit JV, Janssen ML, Schulze H, Jahanshahi A, Van Overbeeke JJ, Temel Y, et al. Deep brain stimulation in tinnitus: current and future perspectives. Brain Res. 2015;1608:51-65.

39. van Zwieten G, Smit JV, Jahanshahi A, Temel Y, Stokroos RJ. Tinnitus: Is there a place for brain stimulation? Surg Neurol Int. 2016;7(Suppl 4):125-9.

40. Rammo R, Ali R, Pabaney A, Seidman M, Schwalb J. Surgical Neuromodulation of Tinnitus: A Review of Current Therapies and Future Applications. Neuromodulation. 2018.

41. Arts RA, George EL, Griessner A, Zierhofer C, Stokroos RJ. Tinnitus Suppression by Intracochlear Electrical Stimulation in Single-Sided Deafness: A Prospective Clinical Trial - Part I. Audiol Neurootol. 2015;20(5):294-313.

42. Tan S, Vlamings R, Lim L, Sesia T, Janssen ML, Steinbusch HW, et al. Experimental deep brain stimulation in animal models. Neurosurgery. 2010;67(4):1073-9. discussion80.

43. Ackermans L, Duits A, van der Linden C, Tijssen M, Schruers K, Temel Y, et al. Double-blind clinical trial of thalamic stimulation in patients with Tourette syndrome. Brain. 2011;134(Pt 3):832-44.

44. Janssen ML, Zwartjes DG, Temel Y, van Kranen-Mastenbroek V, Duits A, Bour LJ, et al. Subthalamic neuronal responses to cortical stimulation. Mov Disord. 2012;27(3):435-8.

45. Joustra J, Buwalda J, Cima R, Free RH, Hofman R, De Kleine E, et al. Richtlijn Tinnitus. Nederlandse Vereniging voor Keel Neus -Oorheelkunde en Heelkunde van het Hoofd - Halsgebied. 2016.

46. Hallam RS, Jakes SC, Hinchcliffe R. Cognitive variables in tinnitus annoyance. Br J Clin Psychol. 1988;27(Pt 3):213-22.

47. Meikle MB, Henry JA, Griest SE, Stewart BJ, Abrams HB, McArdle R, et al. The tinnitus functional index: development of a new clinical measure for chronic, intrusive tinnitus. Ear Hear. 2012;33(2):153-76.

Page $12 / 14$ 
48. Rabau S, Wouters K, Van de Heyning P. Validation and translation of the Dutch tinnitus functional index. B-ENT. 2014;10(4):251-8.

49. Cline ME, Herman J, Shaw ER, Morton RD. Standardization of the visual analogue scale. Nurs Res. 1992;41(6):378-80.

50. Adamchic I, Langguth B, Hauptmann C, Tass PA. Psychometric evaluation of visual analog scale for the assessment of chronic tinnitus. Am J Audiol. 2012;21(2):215-25.

51. Schaper F, Zhao Y, Janssen MLF, Wagner GL, Colon AJ, Hilkman DMW, et al. Single-Cell Recordings to Target the Anterior Nucleus of the Thalamus in Deep Brain Stimulation for Patients with Refractory Epilepsy. Int J Neural Syst. 2018:1850012.

52. Sherman SM. Thalamic relay functions. Prog Brain Res. 2001;134:51-69.

53. Schwartze M, Farrugia N, Kotz SA. Dissociation of formal and temporal predictability in early auditory evoked potentials. Neuropsychologia. 2013;51(2):320-5.

54. Schwartze M, Tavano A, Schroger E, Kotz SA. Temporal aspects of prediction in audition: cortical and subcortical neural mechanisms. Int J Psychophysiol. 2012;83(2):200-7.

55. Wilkinson MD, Dumontier M, Aalbersberg IJ, Appleton G, Axton M, Baak A, et al. The FAIR Guiding Principles for scientific data management and stewardship. Sci Data. 2016;3:160018.

56. Koek RJ, Langevin JP, Krahl SE, Kosoyan HJ, Schwartz HN, Chen JW, et al. Deep brain stimulation of the basolateral amygdala for treatment-refractory combat post-traumatic stress disorder (PTSD): study protocol for a pilot randomized controlled trial with blinded, staggered onset of stimulation. Trials. 2014;15:356.

57. Cheung SW, Racine CA, Henderson-Sabes J, Demopoulos C, Molinaro AM, Heath S, et al. Phase I trial of caudate deep brain stimulation for treatment-resistant tinnitus. J Neurosurg. 2019:1-10.

58. Smit JV, Pielkenrood BJ, Arts R, Janssen ML, Temel Y, Stokroos RJ. Patient Acceptance of Invasive Treatments for Tinnitus. Am J Audiol. 2018;27(2):184-96.

59. Grant RA, Halpern $\mathrm{CH}$, Baltuch GH, O'Reardon JP, Caplan A. Ethical considerations in deep brain stimulation for psychiatric illness. J Clin Neurosci. 2014;21(1):1-5.

\section{Figures}




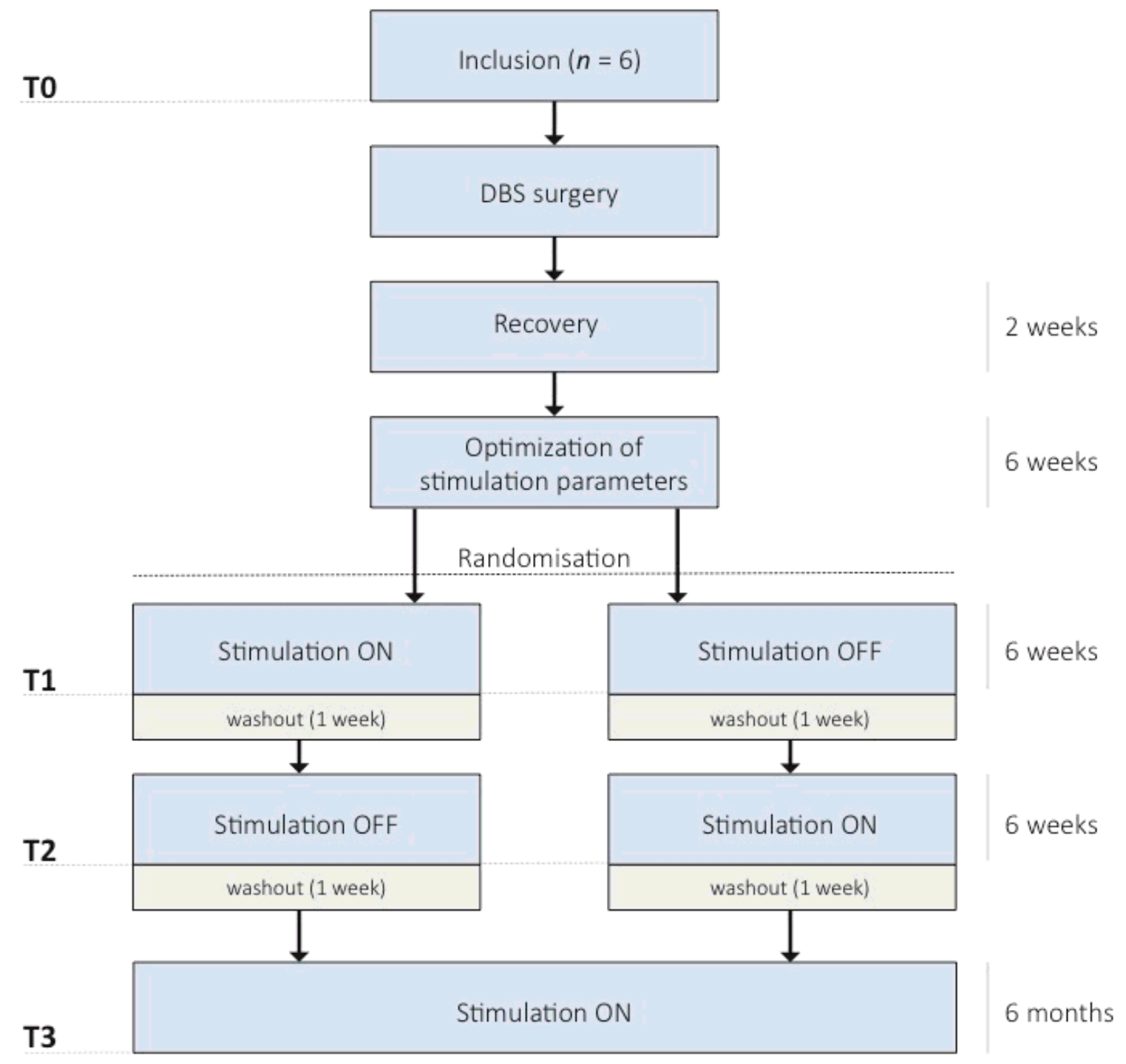

\section{Figure 1}

Overview of study design. 